\title{
Habilidad combinatoria en función de la endogamia para producción y peso del fruto en Cucurbita moschata Duch. ex Poir.
}

\section{Combining ability in the function of inbreeding for yield and average fruit weight in Cucurbita moschata Duch. ex Poir.}

JAVIER ALONSO RESTREPO-SALAZAR ${ }^{1,3}$

FRANCO ALIRIO VALLEJO-CABRERA ${ }^{1}$ EDWIN FERNANDO RESTREPO-SALAZAR ${ }^{2}$

Fruto del híbrido entre líneas endogámicas S2 (Unapal-Abanico-75-1 × Unapal-Llanogrande-2).

Foto: J.A. Restrepo-Salazar

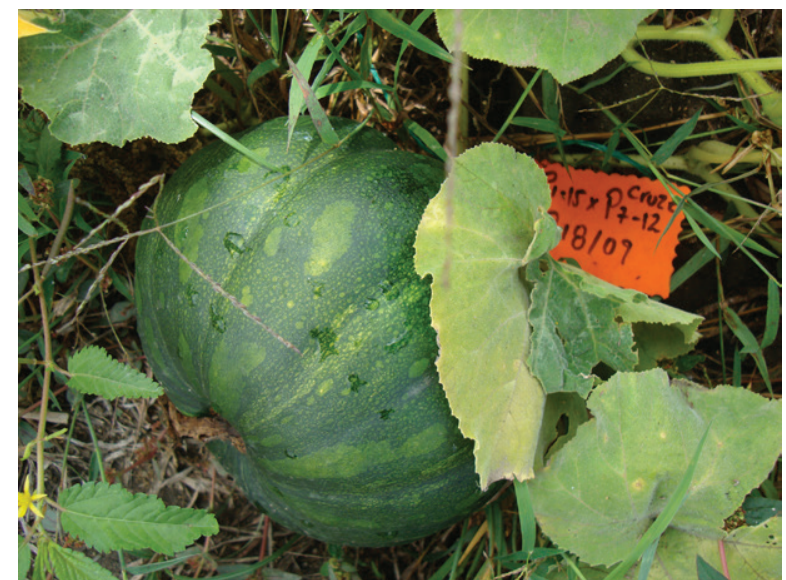

\section{RESUMEN}

Para estimar las habilidades combinatorias general (HCG) y específica (HCE) en función de la endogamia, se evaluaron tres cruzamientos dialélicos de zapallo C. moschata Duch. ex Poir., constituidos cada uno por seis progenitores con tres niveles de endogamia $\left(S_{0}, S_{1}\right.$ y $\left.S_{2}\right)$. Se utilizó un diseño anidado de dos etapas con cuatro repeticiones. Se analizaron las variables producción de frutos por planta (PFP) y peso promedio del fruto (PPF). En el nivel de endogamia $\mathrm{S}_{0}$, solamente la HCG fue importante en la expresión de PFP y PPF. En el nivel de endogamia $S_{1}$, la HCG y HCE fueron importantes en el control genético de PFP y PPF. En el nivel de endogamia $\mathrm{S}_{2}$, la HCG y HCE fueron importantes en la expresión de PPF. Los genotipos más indicados para mejorar PPF con destino al mercado de consumo en fresco, constituido por consumidores que prefieren frutos enteros y no en rodajas, son el progenitor $\mathrm{S}_{0}$ UNAPAL-Dorado (por selección recurrente intrapoblacional) y el híbrido entre líneas endogámicas $S_{2}$ UNAPAL-Abanico-75-1 × UNAPAL-Llanogrande-2 (por selección recurrente recíproca). Igualmente, los genotipos recomendados para mejorar PPF para el mercado de consumo en fresco o uso agroindustrial, conformado por consumidores en los cuales el PPF no es una característica limitante para su adquisición, son la línea endogámica $\mathrm{S}_{2}$ de UNAPAL-Abanico-75-2 (por selección recurrente intrapoblacional), el híbrido entre líneas endogámicas $S_{1}$ UNAPAL-Abanico-75-1 × UNAPAL-Llanogrande-1 y el híbrido entre líneas endogámicas $S_{2}$ UNAPAL-Abanico-75-1 × UNAPAL-Dorado (por selección recurrente recíproca).

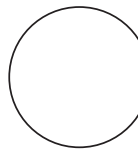

Palabras clave adicionales: zapallo, consumo en fresco, uso agroindustrial cruzamiento dialélico, líneas endogámicas, híbridos.

Facultad de Ciencias Agropecuarias, Universidad Nacional de Colombia, Palmira (Colombia). ORCID Restrepo-Salazar, J.A.: 0000-0002-0293-0709; ORCID Vallejo-Cabrera, F.A.: 0000-0002-2739-0745

2 Escuela de Ingenierías, Universidad Pontificia Bolivariana, Seccional Palmira (Colombia). ORCID Restrepo-Salazar, E.F.: 0000-0002-0408-3312

Autor para correspondencia. jarestrepos@unal.edu.co 


\section{ABSTRACT}

In order to estimate the general and specific combining ability (GCA and SCA) in the function of inbreeding, three diallel crossings of butternut squash $C$. moschata Duch. ex Poir. were evaluated, each coming from six parents with three levels of inbreeding $\left(S_{0}, S_{1}, S_{2}\right)$. A two-stage nested design was used with four replicates. Yield per plant (YPP) and average fruit weight (AFW) were analyzed. In the So generation, only the GCA effects were important in the expression of YPP and AFW. In the first generation of inbreeding $\left(\mathrm{S}_{1}\right)$, both the GCA and SCA effects were important in the genetic control of YPP and AFW. In the second generation of inbreeding $\left(\mathrm{S}_{2}\right)$, both the GCA and SCA effects were again significant in the expression of AFW. The UNAPAL-Dorado $\left(\mathrm{S}_{0}\right.$ parent $)$ and UNAPAL-Abanico-75-1 × UNAPAL-Llanogrande-2 (hybrid of $S_{2}$ inbred lines) are the most indicated genotypes for improving the AFW with intra-population recurrent and reciprocal recurrent selection, respectively, with the target market of fresh consumption by consumers who prefer to buy whole fruits and not sliced fruits. Similarly, the recommended genotypes for improving the AFW for the fresh consumption market and for agro-industrial use by consumers, for whom the weight of the fruit is not a limiting trait for acquisition, include UNAPAL-Abanico-75-2 ( $\mathrm{S}_{2}$ inbred line) with intra-population recurrent selection, UNAPAL-Abanico-75-1 $\times$ UNAPAL-Llanogrande-1 (hybrid of $\mathrm{S}_{1}$ inbred lines) and UNAPAL-Abanico-75-1 $\times$ UNAPAL-Dorado (hybrid of $\mathrm{S}_{2}$ inbred lines), both with reciprocal recurrent selection.

Additional key words: butternut squash, fresh consumption, agro-industrial use, diallel crossing, inbred lines, hybrids.

Fecha de recepción: 09-07-2017 Aprobado para publicación: 30-01-2018

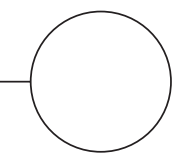

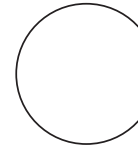

Cucurbita moschata (Duch. ex Lam.) Duch. ex Poir. es la especie domesticada del género Cucurbita más cultivada y consumida en varios departamentos de Colombia. Esta especie es un cultivo importante para la seguridad alimentaria (Restrepo y Vallejo, 2008), pues posee entre otras bondades un alto valor nutritivo debido a la presencia de $\beta$-caroteno (provitamina $A$ ), carotenos totales, ácido ascórbico, carbohidratos, proteínas y minerales (Restrepo, 2015).

En la especie C. moschata no existe un consenso acerca del tipo de acción génica predominante en la expresión y control genético de la variable PFP en cruzamientos dialélicos. En cruzamientos entre progenitores $\mathrm{S}_{0}$, Espitia et al. (2006) y Ortiz et al. (2013) reportan acción génica aditiva; Ruiz et al. (2004) y Toro (2009) sustentan acción génica no aditiva; Ortiz et al. (2013) y Nisha y Veeraragavathatham (2014) documentaron que ambos efectos fueron importantes. En cruzamientos dialélicos entre líneas endogámicas $S_{1}$ se ha reportado efectos aditivos y no aditivos (de los genes) como responsables de la expresión y control genético de PFP (Mohanty, 2000; Espitia et al., 2006; Jha et al., 2009; Pandey et al., 2010; Ortiz et al., 2013; El-Tahawey et al., 2015; Begum et al., 2016), mientras que Rana et al.
(2015) sustenta sólo acción génica no aditiva. En cruzamientos dialélicos entre líneas endogámicas $S_{2}$, también se ha registrado la importancia de ambos efectos en la expresión de PFP (Ortiz et al., 2013).

Para PPF en C. moschata tampoco existe un consenso sobre el tipo de acción génica predominante en la expresión y control genético de la variable. En cruzamientos entre progenitores $\mathrm{S}_{0}$, Gwanama et al. (2001) y Espitia (2004) hallaron efectos aditivos; Ruiz et al. (2004) y Nisha y Veeraragavathatham (2014) sustentaron efectos aditivos y no aditivos. En cruzamientos dialélicos entre líneas endogámicas $S_{1}$, Espitia (2004), Jha et al. (2009), El-Tahawey et al. (2015) y Begum et al. (2016) detectaron efectos aditivos y no aditivos; mientras que Mohanty (2000) reportó solo efectos no aditivos de los genes responsables en la expresión de PPF.

Los objetivos específicos de este estudio fueron: a) estimar los efectos de habilidad combinatoria general y específica en la expresión de la producción por planta y el peso promedio del fruto, en tres cruzamientos dialélicos de Cucurbita moschata, cada uno con diferente grado de endogamia $\left(S_{0}, S_{1}, S_{2}\right)$. b) Identificar 
progenitores o híbridos $F_{1}$ sobresalientes por sus efectos de habilidad combinatoria y por el comportamiento promedio de sus variables.

\section{MATERIALES Y MÉTODOS}

La investigación se realizó en el Centro Experimental de la Universidad Nacional de Colombia, sede Palmira (CEUNP), localizado en Candelaria, Valle del Cauca (Colombia), con coordenadas $3^{\circ} 25^{\prime} 34,42^{\prime \prime} \mathrm{N}$ y $76^{\circ} 25^{\prime} 47,57^{\prime \prime} \mathrm{W}, 980 \mathrm{msnm}, 26^{\circ} \mathrm{C}$ de temperatura promedia anual, $1.100 \mathrm{~mm}$ de precipitación promedia anual y $76 \%$ de humedad relativa media.

Se evaluaron tres cruzamientos dialélicos de zapallo C. moschata, conformados cada uno por seis progenitores con diferentes niveles de endogamia (progenitores a libre polinización $\mathrm{S}_{0}$, líneas endogámicas $\mathrm{S}_{1} \mathrm{y}$ $S_{2}$, derivadas de los progenitores $S_{0}$ ). Los progenitores $\mathrm{S}_{0}$ fueron: UNAPAL-Abanico-75-1, UNAPAL-Abanico-75-2, UNAPAL-Dorado, IC3A, UNAPAL-Llanogrande-1 y UNAPAL-Llanogrande-2, los cuales fueron seleccionados por sus características sobresalientes tamaño y color externo del fruto, color y grosor de la pulpa y origen geográfico diverso. Los frutos fueron seleccionados utilizando un índice de selección ponderado, en el cual se incluyeron las variables peso promedio del fruto $(2,0-4,0 \mathrm{~kg})$, grosor de pulpa $(3,5-5,0$ $\mathrm{cm}$ ) y color salmón de la pulpa. En cada cruzamiento dialélico se evaluaron 21 genotipos (seis progenitores y 15 cruzamientos directos).

La evaluación agronómica de los tres cruzamientos dialélicos se realizó de manera conjunta en un solo experimento durante el segundo semestre de 2011. Se utilizó un diseño anidado de dos etapas con cuatro repeticiones. Se evaluaron dos factores: factor generaciones (factor A) y factor genotipos (factor B). El factor A conformado por tres niveles (generación $\mathrm{S}_{0}$, generación $S_{1}$ y generación $S_{2}$ ) y el factor $B$ constituido por 63 niveles (21 genotipos $S_{0}+21$ genotipos $\mathrm{S}_{1}+21$ genotipos $\mathrm{S}_{2}$ ). Los 21 genotipos $\mathrm{S}_{0}$ quedaron anidados bajo el nivel generación $S_{0}, 21$ genotipos $S_{1}$ quedaron anidados bajo el nivel generación $S_{1}$ y 21 genotipos $\mathrm{S}_{2}$ quedaron anidados bajo el nivel generación $\mathrm{S}_{2}$. La distancia de siembra fue de $2,5 \times 3,0 \mathrm{~m}$ entre surcos y entre plantas, respectivamente. La parcela experimental consistió de un surco de cinco plantas $(37,5$ $\mathrm{m}^{2}$ ). Como parcela útil se utilizaron las tres plantas centrales. Se evaluaron las variables producción de frutos por planta (PFP en $\mathrm{kg}$ ) y peso promedio del fruto (PPF en kg).
El análisis genético y estadístico para estimar la habilidad combinatoria de los diferentes genotipos, se realizó empleando el método propuesto por Hallauer y Miranda (Hallauer et al., 2010). El modelo estadístico fijo asociado al diseño experimental fue:

$$
\mathrm{Y}_{\mathrm{ijkl}}=\mu+\mathrm{g}_{\mathrm{i}}+\mathrm{g}_{\mathrm{j}}+\mathrm{S}_{\mathrm{ij}}+b_{\mathrm{k}}+\mathrm{D}_{1}+(\mathrm{Db})_{\mathbb{l k}}+(1 / \mathrm{rn}) \Sigma \Sigma \mathrm{e}_{\mathrm{ijkl}}
$$

\section{Donde:}

$i, j: 1,2 \ldots \ldots . . p$ padres; $p=6$;

$\mathrm{k}: \quad 1,2 \ldots . . \mathrm{r}$ repeticiones; $\mathrm{r}=4$;

$\mu$ : efecto de la media general del experimento;

$\mathrm{g}_{\mathrm{i}}$ : efecto de habilidad combinatoria general del progenitor "i";

$g_{\mathrm{j}}$ : efecto de habilidad combinatoria general del progenitor " $j$ ";

$S_{\mathrm{ij}}$ : efecto de habilidad combinatoria específica del híbrido entre los progenitores "i" x "j";

$b_{k}$ : efecto del bloque $k$;

$\mathrm{D}_{1}$ : efecto de la generación de endogamia 1 ;

(Db)lk : efecto de la interacción de la generación de endogamia por bloques;

$(1 / \mathrm{rn}) \Sigma \Sigma \mathrm{e}_{\mathrm{ijk} \mid \mathrm{l}}$ : error experimental asociado con la observación Yijkl;

$Y_{i j k l}$ : valor promedio fenotípico observado de la variable en estudio para el cruzamiento (ij) en la generación de endogamia 1.

El análisis de varianza y la estimación de los efectos genéticos para las variables de interés, se realizaron mediante el uso de los programas computacionales SAS ${ }^{\circledR}$ (paquete SAS/STAT ${ }^{\circledR}$, versión 9.4 del sistema SAS ${ }^{\circledR}$ para Windows ()$\left._{)}\right)$de SAS Institute Inc.(C) (2012) y GENES (versión Windows@ $@$, 2004.2.1) de Cruz (2013). Para algunas fuentes de variación del análisis de varianza se utilizó la prueba F y para los efectos genéticos la prueba de T de Student.

\section{RESULTADOS Y DISCUSIÓN}

\section{Análisis de varianza}

Se observaron diferencias significativas entre las generaciones de endogamia $\mathrm{S}_{0}, \mathrm{~S}_{1}$ y $\mathrm{S}_{2}$ de $C$. moschata para las variables producción por planta (PFP) y peso promedio del fruto (PPF), indicando que al menos una de las generaciones resultó ser significativamente diferente del resto (Tab. 1). Resultados similares fueron 
publicados en generaciones de endogamia $\mathrm{S}_{0}, \mathrm{~S}_{1}$ y $\mathrm{S}_{2}$ de C. moschata para PFP (Ortiz et al., 2013). Igualmente, se detectaron diferencias significativas en la fuente de variación genotipos en cada una de las tres generaciones endogámicas evaluadas, y por ende, se puede inferir que existe al menos un padre o un híbrido que presentó un promedio de PFP y PPF diferente estadísticamente del resto de promedios en cada una de dichas generaciones (Tab. 1). Resultados similares fueron reportados en C. moschata para PFP (Espitia et al., 2006) y PPF (Espitia, 2004) en generaciones de endogamia $\mathrm{S}_{0} \mathrm{y}_{1}$.

En la fuente de variación progenitores no se encontraron diferencias estadísticas en la generación de endogamia $\mathrm{S}_{0}$ para PFP y PPF, lo cual se debió posiblemente a que en este trabajo se utilizó un índice de selección que incluyó entre otras variables el PPF, de tal manera que se seleccionaron frutos de los seis progenitores $\mathrm{S}_{0}$ con valores similares de esta variable $(2,0-4,0 \mathrm{~kg})$.
Tabla 1. Cuadrados medios (CM) del ANDEVA para las variables producción de frutos por planta (PFP) y peso promedio del fruto (PPF) en tres generaciones de endogamia de C. moschata, según el método de Hallauer y Miranda (Hallauer et al., 2010).

\begin{tabular}{|c|c|c|c|}
\hline \multirow{3}{*}{ Fuentes de variación } & \multirow{3}{*}{$\mathrm{GI}$} & \multicolumn{2}{|c|}{ Variables } \\
\hline & & PFP (kg) & $\operatorname{PPF}(\mathrm{kg})$ \\
\hline & & $\mathrm{CM}$ & $\mathrm{CM}$ \\
\hline Repeticiones ( R) & 3 & 3,47 & 0,73 \\
\hline Generaciones (D) & 2 & $34,60 *$ & $7,56 * *$ \\
\hline $\mathrm{R}^{*} \mathrm{D}$ & 6 & 10,18 & $1,02 *$ \\
\hline Genotipos(G) & 60 & $57,24 * *$ & $3,28 * *$ \\
\hline Genotipos ( $\left.\mathrm{G} \mathrm{S}_{0}\right)$ & 20 & $26,59 * *$ & $1,51 * *$ \\
\hline Progenitores $\left(\mathrm{P}_{0}\right)$ & 5 & 14,22 & 1,20 \\
\hline Cruzas $\left(\mathrm{C}_{0}\right)$ & 14 & $16,87^{*}$ & $1,34^{* *}$ \\
\hline HCG & 5 & $28,23 *$ & $3,18 * *$ \\
\hline HCE & 9 & 10,56 & 0,31 \\
\hline $\mathrm{P}_{0}$ vs. $\mathrm{C}_{0}$ & 1 & $224,54 * *$ & $5,55^{* *}$ \\
\hline Genotipos $\left(\mathrm{G} \mathrm{S}_{1}\right)$ & 20 & $61,43 * *$ & $3,71 * *$ \\
\hline Progenitores $\left(\mathrm{P}_{1}\right)$ & 5 & $31,62 * *$ & $1,81 *$ \\
\hline Cruzas $\left(\mathrm{C}_{1}\right)$ & 14 & $44,23 * *$ & $2,85 *$ \\
\hline HCG & 5 & $41,35 * *$ & $3,89 * *$ \\
\hline HCE & 9 & $45,83 * *$ & $2,27 * *$ \\
\hline $\mathrm{P}_{1}$ vs. $\mathrm{C}_{1}$ & 1 & $451,29 * *$ & $25,13^{* *}$ \\
\hline Genotipos $\left(\mathrm{G} \mathrm{S}_{2}\right)$ & 20 & $83,70^{* *}$ & $4,62 * *$ \\
\hline Progenitores $\left(\mathrm{P}_{2}\right)$ & 5 & 21,92 & $1,15 *$ \\
\hline Cruzas $\left(\mathrm{C}_{2}\right)$ & 14 & $32,21 * *$ & $2,44 * *$ \\
\hline $\mathrm{HCG}$ & 5 & $58,79 * *$ & $4,62 * *$ \\
\hline HCE & 9 & 17,42 & $1,22 * *$ \\
\hline $\mathrm{P}_{2}$ vs. $\mathrm{C}_{2}$ & 1 & $1113,43^{* *}$ & $52,55 * *$ \\
\hline Error & 180 & 9,63 & 0,40 \\
\hline Media & & 12,28 & 3,39 \\
\hline CV\% & & 25,27 & 18,72 \\
\hline
\end{tabular}

* * **: Significativos al 5 y 1\% de probabilidad, respectivamente, según la prueba de F. HCG: habilidad combinatoria general; HCE: habilidad combinatoria específica; CV: coeficiente de variación. 
Este rango de selección corresponde a valores óptimos de PPF para la comercialización de frutos dirigidos al mercado de consumo en fresco conformado por consumidores que prefieren comprar el fruto entero y no en rodajas (Tab. 1). Estos resultados contrastan con los registrados en generaciones de endogamia $S_{0}$ de C. moschata para PFP (Espitia et al., 2006; Ortiz et al., 2013) y PPF (Espitia, 2004). En la generación de endogamia $S_{1}$ si se presentaron diferencias significativas entre progenitores para PFP y PPF (Tab. 1). Resultados similares fueron reportados en generaciones de endogamia $S_{1}$ de C. moschata para PFP (Ortiz et al., 2013) y PPF (Espitia, 2004); mientras que diferencias no significativas fueron publicadas para PFP por Espitia et al. (2006). En la generación $S_{2}$ solo se registraron diferencias para PPF (Tab. 1); mientras que Ortiz et al. (2013), si registraron diferencias para PFP.

La significancia estadística de los cuadrados medios de PFP y PPF para la fuente de variación cruzas, en las tres generaciones, demuestran que al menos uno de los cruzamientos entre progenitores $S_{0}$ o entre líneas $S_{1}$, o entre líneas $S_{2}$, presentó comportamiento promedio de PFP o PPF diferente del resto (Tab. 1). De lo anterior se infiere que independientemente del nivel de endogamia, es probable encontrar al menos un híbrido con un promedio de PFP o PPF significativamente diferente del resto.

La habilidad combinatoria general (HCG) en la generación de endogamia $S_{0}$ fue significativa para PFP y PPF; sin embargo, la habilidad combinatoria específica (HCE) no fue significativa. La HCG y HCE en la generación de endogamia $S_{1}$, fueron altamente significativas para PFP y PPF. En la generación $\mathrm{S}_{2}$, la HCG fue altamente significativa para PFP y PPF; mientras que la HCE solo fue estadísticamente significativa para PPF. Para el contraste padres vs cruzas (P vs. C), en las tres generaciones de endogamia, se observaron diferencias altamente significativas en PFP y PPF, indicando que el comportamiento promedio de todos los cruzamientos $F_{1}$ (entre progenitores $S_{0}$ y entre líneas $S_{1} \circ S_{2}$ ) fue superior al comportamiento promedio de los progenitores en conjunto, señalando efectos heteróticos significativos en los tres cruzamientos dialélicos para PFP y PPF (Tab. 1). Resultados similares fueron reportados en generaciones $S_{0}$ y $S_{1}$ de $C$. moschata para PFP y PPF (Espitia et al., 2006; Espitia, 2004) y en generaciones $S_{0}, S_{1}$ y $S_{2}$ para PFP (Ortiz et al., 2013).

En el cruzamiento dialélico entre progenitores $\mathrm{S}_{0}$ solo los efectos aditivos (HCG) fueron importantes en la expresión genética de PFP (Tab. 1). Resultados similares fueron reportados en C. moschata por, Espitia et al. (2006) y Ortiz et al. (2013), quienes encontraron que la acción génica aditiva fue importante en la expresión genética de PFP en cruzamientos dialélicos entre progenitores $S_{0}$. En contraste, en cruzamientos dialélicos entre progenitores $S_{0}$ de C. moschata se ha publicado que sólo la acción génica no aditiva fue importante (Ruiz et al., 2004; Toro, 2009). Por otro lado, en esta especie en un cruzamiento dialélico entre progenitores $S_{0}$ para PFP se ha registrado que ambos efectos fueron importantes (Ortiz et al., 2013; Nisha y Veeraragavathatham, 2014).

En el cruzamiento dialélico entre líneas endogámicas $\mathrm{S}_{1}$ la HCG y HCE, indicaron la importancia de la acción génica aditiva y no aditiva en la expresión y control genético de la PFP (Tab. 1). Resultados similares fueron registrados por Mohanty (2000), Espitia et al. (2006), Jha et al. (2009), Pandey et al. (2010), Ortiz et al. (2013), El-Tahawey et al. (2015) y Begum et al. (2016) en C. moschata, los cuales encontraron que los efectos aditivos y no aditivos de los genes fueron responsables de la expresión de PFP en cruzamientos dialélicos entre líneas endogámicas $\mathrm{S}_{1}$. Contrariamente, Rana et al. (2015) publicaron que sólo la acción génica no aditiva fue la importante en la expresión de PFP.

En el cruzamiento dialélico entre líneas endogámicas $\mathrm{S}_{2}$ solo los efectos aditivos (HCG) fueron importantes para PFP (Tab. 1). Estos resultados contrastan con los registrados por Ortiz et al. (2013), quienes observaron que ambos efectos fueron estadísticamente significativos para PFP en cruzamientos dialélicos entre líneas endogámicas $\mathrm{S}_{2}$ de C. moschata.

En el cruzamiento dialélico entre progenitores $S_{0}$ de C. moschata para la expresión y control genético del PPF solo los efectos genéticos aditivos fueron importantes (Tab. 1). Resultados similares fueron reportados por Gwanama et al. (2001) y Espitia (2004) en C. moschata, quienes encontraron que solo los efectos aditivos fueron importantes para esta variable en el cruzamiento dialélico entre progenitores $\mathrm{S}_{0}$. En contraste, para PPF también se ha reportado la importancia de los efectos aditivos y no aditivos (Ruiz et al., 2004; Nisha y Veeraragavathatham, 2014).

En los cruzamientos dialélicos entre líneas endogámicas $\mathrm{S}_{1}$ y $\mathrm{S}_{2}$ la HCG y HCE fueron importantes en PPF, indicando la importancia de la acción génica aditiva y no aditiva en su expresión genética (Tab. 1). Espitia (2004), Jha et al. (2009), El-Tahawey et al. (2015) y Begum et al. (2016), también encontraron en líneas 
endogámicas $\mathrm{S}_{1}$ de C. moschata la importancia de efectos aditivos y no aditivos de los genes en la expresión de PPF. Por otro lado, Mohanty (2000) al realizar un cruzamiento dialélico entre líneas $S_{1}$ en $C$. moschata, reportó solo efectos no aditivos de los genes como los responsables de la expresión de PPF.

Al realizar un análisis global entre generaciones de endogamia se puede deducir, que los efectos aditivos fueron importantes en la expresión de PFP y PPF en todas las generaciones endogámicas evaluadas; mientras que los efectos no aditivos de los genes solo fueron importantes en los cruzamientos dialélicos entre líneas endogámicas en comparación con el cruzamiento dialélico entre progenitores $S_{0}$ para estas variables. Este mismo comportamiento ha sido reportado por Espitia (2004) y Espitia et al. (2006) en C. moschata para esas variables, comparando el cruzamiento dialélico entre progenitores $\mathrm{S}_{0}$ con el cruzamiento dialélico entre líneas endogámicas $S_{1}$. Resultados iguales han sido reportados en maíz por varios autores (Crossa et al., 1990; Rezende y Souza Junior, 2000), los cuales dan como una posible explicación a esto, la mayor divergencia genética que puede presentarse en los cruzamientos entre progenitores de base genética estrecha $\left(\mathrm{S}_{1}\right.$ y $\left.\mathrm{S}_{2}\right)$ comparada con la que se presenta en cruzamientos entre progenitores de base amplia $\left(\mathrm{S}_{0}\right)$.

\section{Heterosis promedia para PFP y PPF}

La heterosis promedia en las generaciones de endogamia $S_{1} y S_{2}$ fue significativa para PFP y PPF; no así para la generación $\mathrm{S}_{0}$ (Tabs. 2 y 3). La mayor expresión de la heterosis promedia detectada en este estudio, en el dialelo entre líneas endogámicas $\mathrm{S}_{1}$ y $\mathrm{S}_{2}$ en comparación con el dialelo de progenitores $\mathrm{S}_{0}$, ha sido explicada por otros autores como Hallauer et al. (2010), los cuales han reportado que las progenies de líneas endogámicas pueden presentar una mayor heterosis con respecto a progenies de poblaciones de base genética amplia, cuando se utilizan como progenitores líneas genéticamente divergentes y se presentan niveles de dominancia unidireccional en la mayoría de loci que codifican por el carácter que se quiere mejorar.

\section{Efectos de habilidad combinatoria para PFP}

Para PFP ninguno de los progenitores $\mathrm{S}_{0}$ o de las líneas endogámicas $S_{1}$ y $S_{2}$, presentaron efectos de HCG significativos (Tab. 2), lo cual fue debido posiblemente a que en este trabajo de investigación la producción de las diferentes generaciones de endogamia estuvo acompañada de selección, donde los frutos seleccionados de los progenitores $\mathrm{S}_{0}$ o de las líneas endogámicas $S_{1}$ y $S_{2}$, tenían pesos promedios intermedios $(2,0-4,0$ $\mathrm{kg}$ ), y por ende, no necesariamente correspondían a individuos con las mayores PFP. Algunas investigaciones reportaron que ningún progenitor $\mathrm{S}_{0}$ presentó efectos de HCG significativos para PFP en C. moschata (Ruiz et al., 2004; Toro, 2009) y en ninguna línea endogámica $S_{1}$ (Rana et al., 2015). Otras investigaciones registraron al menos un progenitor $\mathrm{S}_{0}$ con efectos de HCG significativos (Espitia et al., 2006; Ortiz et al., 2013; Nisha y Veeraragavathatham, 2014). Contrariamente a lo observado en este estudio, Mohanty, (2000), Espitia et al. (2006), Jha et al. (2009), Pandey et al. (2010), Ortiz et al. (2013), El-Tahawey et al. (2015) y Begum et al. (2016) encontraron al menos una línea endogámica $S_{1}$ con efectos de HCG estadísticamente significativos para PFP en C. moschata. Por otro lado, Ortiz et al. (2013) tampoco encontraron líneas endogámicas $\mathrm{S}_{2}$ con efectos de HCG significativos para dicha variable.

Para PFP ningún cruzamiento registró efectos de HCE significativos (Tab. 2). Las causas podrían ser la falta de complementación genética entre los progenitores que participaron en los cruzamientos o la carencia de niveles importantes de divergencia genética, aunque ésta última causa podría ser la menos posible, pues los progenitores $S_{0}$ tienen un origen geográfico diferente (IC3A: Costa Rica-Centroamérica; UNAPAL-Abanico-75-1 y UNAPAL-Abanico-75-2: Magdalena; UNAPAL-Dorado: Valle del Cauca; UNAPAL-Llanogrande-1 y UNAPAL-Llanogrande-2: Cauca). En contraste a lo observado en este estudio, en progenitores $S_{0}$ (Ruiz et al., 2004; Toro, 2009; Nisha y Veeraragavathatham, 2014) y en líneas endogámicas $S_{1}$ (Mohanty, 2000; Espitia et al., 2006; Jha et al., 2009; Pandey et al., 2010; Ortiz et al., 2013; Rana et al., 2015; El-Tahawey et al. 2015; Begum et al., 2016), encontraron al menos un híbrido con efectos de HCE estadísticamente significativos para PFP. Por otro lado, Ortiz et al. (2013) encontraron un híbrido de líneas endogámicas $\mathrm{S}_{2}$ con efectos de HCE estadísticamente significativos para esta variable.

\section{Efectos de habilidad combinatoria para PPF}

El comportamiento diferencial de los progenitores para PPF en sus valores como progenitor $\left(g_{i}\right)$ (Tab. $3)$, confirman las diferencias altamente significativas detectadas para HCG en el ANDEVA en los tres cruzamientos dialélicos (Tab. 1) y favorece la selección 
Tabla 2. Producción por planta (PFP) para progenitores (diagonal), híbridos (arriba de la diagonal), efectos de habilidad combinatoria especifica $\left(s_{i j}\right)$ (debajo de la diagonal), efectos de habilidad combinatoria general $\left(g_{i}\right)$ y promedio de los híbridos de zapallo $C$. moschata, en los cruzamientos dialélicos entre progenitores $S_{0}$ y entre líneas endogámicas $S_{1}$ y $S_{2}$.

\begin{tabular}{|c|c|c|c|c|c|c|c|c|c|}
\hline \multicolumn{10}{|c|}{ Cruzamiento dialélico entre progenitores So } \\
\hline \multicolumn{2}{|r|}{ Progenitores } & \multirow{2}{*}{$\frac{\mathrm{P} 1}{11,14}$} & \multirow{2}{*}{$\frac{\mathrm{P} 2}{10,28}$} & \multirow{2}{*}{$\frac{\text { P3 }}{15,00}$} & \multirow{2}{*}{$\begin{array}{c}\mathrm{P4} \\
13,89\end{array}$} & \multirow{2}{*}{$\begin{array}{c}\mathrm{P5} \\
13,05\end{array}$} & \multirow{2}{*}{$\frac{P 6}{10,71}$} & \multirow{2}{*}{$\frac{\mathrm{Gi}}{-0,06}$} & \multirow{2}{*}{$\frac{\text { Promedio de híbridos }}{12,59}$} \\
\hline P1 & Abanico 75-1 & & & & & & & & \\
\hline P2 & Abanico 75-2 & $-1,55$ & 6,86 & 12,46 & 12,04 & 12,04 & 12,25 & $-0,73$ & 11,81 \\
\hline P3 & Dorado & 0,65 & $-1,23$ & 11,36 & 14,95 & 14,45 & 13,38 & 1,78 & 14,05 \\
\hline P4 & IC-G3A & 0,88 & $-0,30$ & 0,09 & 9,13 & 15,42 & 8,63 & 0,45 & 12,99 \\
\hline P5 & Llanogrande-1 & $-0,22$ & 0,62 & $-0,61$ & 1,67 & 7,82 & 9,71 & 0,67 & 12,93 \\
\hline P6 & Llanogrande-2 & 0,24 & 2,46 & 1,10 & $-2,34$ & $-1,47$ & 7,78 & $-2,11$ & 10,93 \\
\hline & & & & & & \multicolumn{3}{|c|}{ Promedio progenitores } & 9,01 \\
\hline & & & & & & \multicolumn{3}{|c|}{ Promedio híbridos } & 12,55 \\
\hline & & & & & & \multicolumn{3}{|c|}{ Heterosis promedia } & 3,54 \\
\hline
\end{tabular}

Cruzamiento dialélico entre líneas endogámicas $\mathrm{S}_{1}$

\begin{tabular}{|c|c|c|c|c|c|c|c|c|c|}
\hline \multicolumn{2}{|r|}{ Progenitores } & \multirow{2}{*}{$\begin{array}{c}\mathrm{P} 1 \\
8,57 \\
\end{array}$} & \multirow{2}{*}{$\begin{array}{c}\mathrm{P} 2 \\
12,08 \\
\end{array}$} & \multirow{2}{*}{$\begin{array}{c}\text { P3 } \\
12,32 \\
\end{array}$} & \multirow{2}{*}{$\begin{array}{c}\mathrm{P} 4 \\
14,75 \\
\end{array}$} & \multirow{2}{*}{$\begin{array}{c}\text { P5 } \\
20,50 \\
\end{array}$} & \multirow{2}{*}{$\begin{array}{c}\text { P6 } \\
10,33 \\
\end{array}$} & \multirow{2}{*}{$\begin{array}{c}\mathrm{Gi} \\
0,19 \\
\end{array}$} & \multirow{2}{*}{$\begin{array}{c}\text { Promedio de híbridos } \\
\qquad 14,00 \\
\end{array}$} \\
\hline P1 & Abanico 75-1 & & & & & & & & \\
\hline P2 & Abanico 75-2 & $-2,93$ & 13,54 & 17,19 & 13,67 & 15,38 & 14,88 & 0,98 & 14,64 \\
\hline P3 & Dorado & $-2,37$ & 1,72 & 6,54 & 10,13 & 19,08 & 13,08 & 0,62 & 14,35 \\
\hline P4 & IC-G3A & 1,82 & $-0,10$ & $-3,26$ & 10,21 & 13,83 & 12,54 & $-1,09$ & 12,98 \\
\hline P5 & Llanogrande-1 & 4,56 & $-1,33$ & 2,71 & $-0,83$ & 5,80 & 8,03 & 1,89 & 15,36 \\
\hline P6 & Llanogrande-2 & $-1,09$ & 2,64 & 1,20 & 2,36 & $-5,12$ & 7,67 & $-2,60$ & 11,76 \\
\hline & & & & & & \multicolumn{3}{|c|}{ Promedio progenitores } & 8,72 \\
\hline & & & & & & \multicolumn{3}{|c|}{ Promedio híbridos } & 13,85 \\
\hline & & & & & & \multicolumn{3}{|c|}{ Heterosis promedia } & $5,13^{*}$ \\
\hline
\end{tabular}

Cruzamiento dialélico entre Líneas endogámicas $\mathrm{S}_{2}$

\begin{tabular}{|c|c|c|c|c|c|c|c|c|c|}
\hline \multicolumn{2}{|r|}{ Progenitores } & \multirow{2}{*}{$\frac{\mathrm{P} 1}{9,02}$} & \multirow{2}{*}{$\frac{\text { P2 }}{17,88}$} & \multirow{2}{*}{$\frac{\text { P3 }}{20,87}$} & \multirow{2}{*}{$\begin{array}{r}\mathrm{P4} \\
15,33\end{array}$} & \multirow{2}{*}{$\frac{\text { P5 }}{15,00}$} & \multirow{2}{*}{$\frac{\text { P6 }}{14,00}$} & \multirow{2}{*}{$\frac{\mathrm{Gi}}{1,80}$} & \multirow{2}{*}{$\frac{\text { Promedio de híbridos }}{16,61}$} \\
\hline P1 & Abanico 75-1 & & & & & & & & \\
\hline P2 & Abanico 75-2 & $-1,31$ & 8,48 & 16,00 & 16,73 & 15,56 & 18,47 & 2,19 & 16,93 \\
\hline P3 & Dorado & 2,77 & $-2,49$ & 7,85 & 13,73 & 14,98 & 14,81 & 1,14 & 16,08 \\
\hline P4 & IC-G3A & $-0,31$ & 0,70 & $-1,28$ & 8,36 & 14,30 & 10,46 & $-1,33$ & 14,11 \\
\hline P5 & Llanogrande-1 & $-0,34$ & $-0,15$ & 0,32 & 2,09 & 6,25 & 9,47 & $-1,63$ & 13,86 \\
\hline P6 & Llanogrande-2 & $-0,81$ & 3,25 & 0,68 & $-1,20$ & $-1,91$ & 2,75 & $-2,16$ & 13,44 \\
\hline & & & & & & \multicolumn{3}{|c|}{ Promedio progenitores } & 7,12 \\
\hline & & & & & & \multicolumn{3}{|c|}{ Promedio híbridos } & 15,17 \\
\hline & & & & & & \multicolumn{3}{|c|}{ Heterosis promedia } & $8,05^{* *}$ \\
\hline
\end{tabular}

${ }^{*},{ }^{* *}$ : Significativos al $5 \%$ y $1 \%$ de probabilidad, respectivamente, según la prueba de T de Student.

de nuevos genotipos homocigotos o progenitores superiores, dado que los genes con efectos aditivos son los que responden a la selección. Gwanama et al. (2001), Ruiz et al. (2004), Espitia (2004) y Nisha y Veeraragavathatham (2014), también encontraron en
C. moschata al menos un progenitor $\mathrm{S}_{0}$ con valores de gi (efectos de HCG) estadísticamente significativos para PPF. Igualmente, Espitia (2004), Jha et al. (2009) y El-Tahawey et al. (2015) reportaron en C. moschata varias líneas endogámicas $S_{1}$ con efectos de HCG 


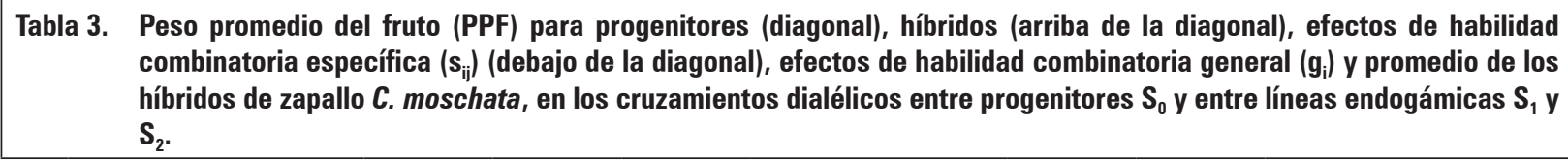

\begin{tabular}{|c|c|c|c|c|c|c|c|c|c|}
\hline \multicolumn{10}{|c|}{ Cruzamiento dialélico entre progenitores $S_{0}$} \\
\hline \multicolumn{2}{|r|}{ Progenitores } & \multirow{2}{*}{$\begin{array}{r}\mathrm{P} 1 \\
2,99\end{array}$} & \multirow{2}{*}{$\begin{array}{r}\mathrm{P} 2 \\
2,62\end{array}$} & \multirow{2}{*}{$\begin{array}{r}\text { P3 } \\
4,02\end{array}$} & \multirow{2}{*}{$\begin{array}{r}\mathrm{P} 4 \\
3,90\end{array}$} & \multirow{2}{*}{$\begin{array}{r}\mathrm{P5} \\
3,16\end{array}$} & \multirow{2}{*}{$\frac{P 6}{2,60}$} & \multirow{2}{*}{$\frac{\mathrm{Gi}}{0,05}$} & \multirow{2}{*}{$\begin{array}{c}\text { Promedio de híbridos } \\
3,26\end{array}$} \\
\hline P1 & Abanico 75-1 & & & & & & & & \\
\hline P2 & Abanico 75-2 & $-0,33^{* *}$ & 1,90 & 3,36 & 3,21 & 2,69 & 3,00 & $-0,31$ & 2,98 \\
\hline P3 & Dorado & 0,11 & $-0,16^{*}$ & 3,22 & 4,28 & 3,53 & 3,37 & $0,62^{*}$ & 3,71 \\
\hline P4 & IC3A & $0,21^{* *}$ & $-0,13$ & 0,02 & 3,09 & 3,40 & 2,95 & $0,41 *$ & 3,55 \\
\hline P5 & Llanogrande-1 & $0,17^{*}$ & 0,05 & 0,00 & 0,05 & 2,15 & 2,17 & $-0,29$ & 2,99 \\
\hline P6 & Llanogrande-2 & $-0,17^{*}$ & $0,57^{* *}$ & 0,04 & $-0,16^{*}$ & $-0,28^{* *}$ & 2,56 & $-0,50^{*}$ & 2,82 \\
\hline & & & & & & \multicolumn{3}{|c|}{ Promedio progenitores } & 2,65 \\
\hline & & & & & & \multicolumn{3}{|c|}{ Promedio híbridos } & 3,22 \\
\hline & & & & & & \multicolumn{3}{|c|}{ Heterosis promedia } & 0,56 \\
\hline
\end{tabular}

\begin{tabular}{|c|c|c|c|c|c|c|c|c|c|}
\hline \multicolumn{10}{|c|}{ Cruzamiento dialélico entre líneas endogámicas $S_{1}$} \\
\hline \multicolumn{2}{|r|}{ Progenitores } & \multirow{2}{*}{$\frac{\mathrm{P} 1}{3,12}$} & \multirow{2}{*}{$\begin{array}{r}\text { P2 } \\
4,19\end{array}$} & \multirow{2}{*}{$\begin{array}{r}\text { P3 } \\
3,64\end{array}$} & \multirow{2}{*}{$\begin{array}{r}\text { P4 } \\
4,15\end{array}$} & \multirow{2}{*}{$\begin{array}{r}\mathrm{P5} \\
5,73\end{array}$} & \multirow{2}{*}{$\begin{array}{r}\mathrm{P} 6 \\
3,20\end{array}$} & \multirow{2}{*}{$\frac{\mathrm{Gi}}{0,28}$} & \multirow{2}{*}{$\frac{\text { Promedio de híbridos }}{4,18}$} \\
\hline P1 & Abanico 75-1 & & & & & & & & \\
\hline P2 & Abanico 75-2 & $-0,71$ & 3,73 & 5,16 & 3,82 & 4,86 & 4,56 & $0,68^{*}$ & 4,52 \\
\hline P3 & Dorado & $-0,56$ & 0,58 & 2,30 & 3,32 & 4,01 & 3,41 & $-0,07$ & 3,91 \\
\hline P4 & IC3A & 0,30 & $-0,46$ & $-0,16$ & 2,30 & 3,49 & 3,49 & $-0,41$ & 3,66 \\
\hline P5 & Llanogrande-1 & $1,31^{* *}$ & 0,03 & $-0,07$ & $-0,28$ & 2,03 & 2,47 & 0,18 & 4,11 \\
\hline P6 & Llanogrande-2 & $-0,35$ & 0,56 & 0,20 & 0,59 & $-1,00^{*}$ & 2,20 & $-0,68^{*}$ & 3,43 \\
\hline & & & & & & \multicolumn{3}{|c|}{ Promedio progenitores } & 2,76 \\
\hline & & & & & & \multicolumn{3}{|c|}{ Promedio híbridos } & 3,97 \\
\hline & & & & & & \multicolumn{3}{|c|}{ Heterosis promedia } & $1,20 * *$ \\
\hline
\end{tabular}

\begin{tabular}{|c|c|c|c|c|c|c|c|c|c|}
\hline \multicolumn{10}{|c|}{ Cruzamiento dialélico entre líneas endogámicas $\mathrm{S}_{2}$} \\
\hline \multicolumn{2}{|r|}{ Progenitores } & \multirow{2}{*}{$\begin{array}{r}\mathrm{P} 1 \\
2,39\end{array}$} & \multirow{2}{*}{$\begin{array}{r}\text { P2 } \\
4,84\end{array}$} & \multirow{2}{*}{$\begin{array}{r}\text { P3 } \\
4,98\end{array}$} & \multirow{2}{*}{$\begin{array}{r}\text { P4 } \\
4,53\end{array}$} & \multirow{2}{*}{$\begin{array}{r}\text { P5 } \\
3,50 \\
\end{array}$} & \multirow{2}{*}{\begin{tabular}{|c|}
$\mathrm{P} 6$ \\
2,89 \\
\end{tabular}} & \multirow{2}{*}{$\begin{array}{r}\mathrm{Gi} \\
0,21\end{array}$} & \multirow{2}{*}{$\begin{array}{c}\text { Promedio de híbridos } \\
4,15\end{array}$} \\
\hline P1 & Abanico 75-1 & & & & & & & & \\
\hline P2 & Abanico 75-2 & $-0,10$ & 2,75 & 4,79 & 4,22 & 4,15 & 4,89 & $0,74^{*}$ & 4,58 \\
\hline P3 & Dorado & $0,49 *$ & $-0,24$ & 2,33 & 3,76 & 3,84 & 3,73 & 0,30 & 4,22 \\
\hline P4 & IC3A & 0,40 & $-0,43$ & $-0,44$ & 2,47 & 4,17 & 2,91 & $-0,08$ & 3,92 \\
\hline P5 & Llanogrande-1 & $-0,23$ & $-0,14$ & $-0,02$ & $0,70^{*}$ & 2,28 & 2,49 & $-0,44$ & 3,63 \\
\hline P6 & Llanogrande-2 & $-0,56^{*}$ & $0,90^{* *}$ & 0,20 & $-0,24$ & $-0,31$ & 1,21 & $-0,74^{*}$ & 3,39 \\
\hline & & & & & & \multicolumn{3}{|c|}{ Promedio progenitores } & 2,24 \\
\hline & & & & & & \multicolumn{3}{|c|}{ Promedio híbridos } & 3,98 \\
\hline & & & & & & \multicolumn{3}{|c|}{ Heterosis promedia } & $1,74^{* *}$ \\
\hline
\end{tabular}

* , **: Significativos al 5\% y $1 \%$ de probabilidad, respectivamente, según la prueba de T de Student.

diferentes de cero para PPF. Por otro lado y con respecto a los cruzamientos dialélicos entre líneas endogámicas $S_{1}$ y $S_{2}$, los resultados de los efectos de HCE (Sij) para PPF (Tab. 3), también confirman los niveles de significancia estadística del ANDEVA (Tab. 1).
Similarmente, Mohanty (2000), Espitia (2004), Jha et al. (2009) y Begum et al. (2016) encontraron en C. moschata algunos híbridos entre líneas endogámicas $\mathrm{S}_{1}$ con efectos de HCE estadísticamente diferentes de cero para PPF. 


\section{Genotipos recomendados para el mejoramiento genético de PPF}

El progenitor más indicado para mejorar PPF con destino al mercado de consumo en fresco constituido por consumidores que prefieren comprar frutos enteros $y$ no en rodajas, mediante el aprovechamiento de los efectos aditivos por selección recurrente intrapoblacional, es el progenitor $\mathrm{S}_{0}$ UNAPAL-Dorado, debido a que presentó un valor en los efectos de HCG significativo $\left(0,62^{* *}\right)$, un promedio en sus híbridos de PPF con un valor óptimo para ser comercializado como fruto entero (3,71 kg) (Tab. 3) y un promedio en sus híbridos de PFP aceptable (14,05 kg/planta) (Tab. 2). Por otro lado, el progenitor recomendado para mejorar PPF, mediante el aprovechamiento de los efectos aditivos y no aditivos por selección recurrente intrapoblacional, dirigido al mercado de consumo en fresco y uso agroindustrial, conformado por aquellos consumidores en los cuales el peso del fruto no es una característica limitante para su adquisición, es la línea endogámica $\mathrm{S}_{2}$ de UNAPAL-Abanico-75-2, pues registró un valor en los efectos de HCG significativo $\left(0,74^{*}\right)$, un promedio en sus híbridos de PPF de 4,58 $\mathrm{kg}$ (Tab. 3) y el promedio en sus híbridos de PFP más alto de todos los progenitores evaluados $(16,93 \mathrm{~kg} /$ planta) (Tab. 2).

El híbrido más indicado para mejorar PPF mediante el aprovechamiento de los efectos aditivos y no aditivos por selección recurrente recíproca, dirigido al mercado de consumo en fresco conformado por consumidores que prefieren comprar frutos enteros y no en rodajas, es el híbrido entre líneas endogámicas $\mathrm{S}_{2}$ UNAPAL-Abanico-75-1 × UNAPAL-Llanogrande-2, debido a que presentó un valor en los efectos de HCE significativo $\left(-0,56^{*}\right)$, un valor óptimo de PPF para ser comercializado como fruto entero $(2,89 \mathrm{~kg})$ (Tab. 3) y un valor de PFP aceptable (14,00 kg/planta) (Tab. 2). Por otro lado, los híbridos recomendados para el mejoramiento de PPF a través de la explotación de los efectos aditivos y no aditivos por selección recurrente recíproca, con destino al mercado de consumo en fresco y uso agroindustrial, constituido por aquellos consumidores en los cuales el peso del fruto no es una característica limitante para su adquisición, son el híbrido entre líneas endogámicas $\mathrm{S}_{1}$ UNAPAL-Abanico-75-1 × UNAPAL-Llanogrande-1 y el híbrido entre líneas endogámicas $S_{2}$ UNAPAL-Abanico-75-1 $\times$ UNAPAL-Dorado. Ambos híbridos presentaron valores en los efectos de HCE significativos $\left(1,31^{* *}\right.$ y $0,49^{*}$, respectivamente), valores de PPF de 5,73 y 4,98 kg, respectivamente (Tab. 3); así como también los valores de PFP más altos entre todos los híbridos evaluados (20,50 y $20,87 \mathrm{~kg} /$ planta, respectivamente) (Tab. 2).

Los valores de los efectos de HCE negativos y significativos para algunos de los híbridos, sugiere que su promedio fue inferior al promedio esperado con base en la gi de sus progenitores y la media general, considerándose por ello como una alternativa genética viable para reducir el tamaño o peso promedio del fruto y así satisfacer las preferencias del mercado fresco de zapallo.

\section{CONCLUSIONES}

En la generación de endogamia $S_{0}$ solo la habilidad combinatoria general fue importante en la expresión de PFP y PPF. En contraste, en la generación de endogamia $S_{1}$ tanto la habilidad combinatoria general como la habilidad combinatoria específica, contribuyeron al control genético de PFP y PPF.

En la generación de endogamia $S_{2}$, las habilidades combinatorias general y específica fueron responsables del control de la expresión del PPF; mientras que en PFP solo la habilidad combinatoria general fue importante.

Los genotipos más indicados para mejorar PPF con destino al mercado de consumo en fresco constituido por consumidores que prefieren comprar frutos enteros y no en rodajas, son el progenitor $\mathrm{S}_{0}$ UNAPAL-Dorado y el híbrido entre líneas endogámicas $S_{2}$ UNAPAL-Abanico-75-1 x UNAPAL-Llanogrande-2.

Los genotipos recomendados para mejorar PPF para el mercado de consumo en fresco y uso agroindustrial, conformado por aquellos consumidores en los cuales el peso del fruto no es una característica limitante para su adquisición, son la línea endogámica $S_{2}$ de UNAPAL-Abanico-75-2, el híbrido entre líneas endogámicas $S_{1}$ UNAPAL-Abanico-75-1 × UNAPAL-Llanogrande-1 y el híbrido entre líneas endogámicas $S_{2}$ UNAPAL-Abanico-75-1 × UNAPAL-Dorado.

\section{AGRADECIMIENTOS}

Al Programa Mejoramiento Genético, Agronomía y Producción de Semillas de Hortalizas de la Universidad Nacional de Colombia-Sede Palmira. Al 
Departamento Administrativo de Ciencia, Tecnología e Innovación-COLCIENCIAS de Colombia.

Conflicto de intereses: el manuscrito fue preparado y revisado con la participación de los autores, quienes declaran no tener algún conflicto de interés que coloquen en riesgo la validez de los resultados aquí presentados.

\section{REFERENCIAS BIBLIOGRÁFICAS}

Begum, F., A.M. Akanda, M.A.T. Masud, M.D.G. Rasul y M.A. Islam. 2016. Combining ability and heterosis for PRSV-W resistance in pumpkin (Cucurbita moschata). J. Int. Acad. Res. Multidisciplinary 4(1), 92-102.

Crossa, J., S.K. Vasal y D.L. Beck. 1990. Combining ability in diallel crosses of CYMMYT's tropical late yelow maize germplasm. Maydica 35, 273-278.

Cruz, C.D. 2013. GENES - a software package for analysis in experimental statistics and quantitative genetics. Acta Scient. Agron. 35(3), 271-276. Doi: 10.4025/actasciagron.v35i3.21251

El-Tahawey, M.A.F.A., A.M. Kandeel, S.M.S. Youssef y M.M.M. Abd El-Salam. 2015. Heterosis, potence ratio, combining ability and correlation of some economic traits in diallel crosses of pumpkins. Egyp. J. Plant Breed. 19(2), 419-439. Doi: 10.12816/0011721

Espitia, M. 2004. Estimación y análisis de parámetros genéticos en cruzamientos dialélicos de zapallo (Cucurbita moschata Duch. ex Poir.). Tesis de doctorado. Facultad de Ciencias Agropecuarias, Universidad Nacional de Colombia, Palmira, Colombia.

Espitia, C.M., C.F.A. Vallejo y G.D. Baena. 2006. Efectos heteróticos y habilidad combinatoria para el rendimiento por planta en Cucurbita moschata Duch. Ex Poir. Rev. Fac. Nac. Agron. Medellín 59(1), 3105-3121.

Gwanama, C., A.M. Botha y M.T. Labuschane. 2001. Genetic effects and heterosis of flowering and fruit characteristic of tropical pumpkin. Plant Breed. 120(3), 271-272. Doi: 10.1046/j.1439-0523.2001.00595.x

Hallauer, A.R., M.J. Carena y J.B. Miranda. 2010. Quantitative genetics in maize breeding. Springer, New York, USA. Doi: 10.1007/978-1-4419-0766-0

Jha, A., S. Pandey, M. Rai, D.S. Yadav y T.B. Singh. 2009. Heterosis in relation to combining ability for flowering behaviors and yield parameters in pumpkin. Veget. Sci. 36(3), 332-335.

Mohanty, B.K. 2000. Combining ability for yield and its components in pumpkin. Indian J. Genet. Plant Breed. 60(3), 373-379.

Nisha, S.K. y D. Veeraragavathatham. 2014. Heterosis and combining ability for fruit yield and its component traits in pumpkin (Cucurbita moschata Duch. ex Poir.). Adv. Appl. Res. 6(2), 158-162. Doi: 10.5958/2349-2104.2014.00008.4

Ortiz, G.S., F.A. Vallejo, D. Baena, E.I. Estrada y M.P. Valdés. 2013. Zapallo para consumo en fresco y fines agroindustriales: investigación y desarrollo. Universidad $\mathrm{Na}$ cional de Colombia, Palmira, Colombia.

Pandey, S., A. Jha, S. Kumar y M. Rai. 2010. Genetics and heterosis of quality and yield of pumpkin. Ind. J. Hortic. 67(3), 333-338.

Rana, M.S., M.G. Rasul, A.K.M.A. Islam y M.M. Hossain. 2015. Combining ability of quality traits in pumpkin (Cucurbita moschata Duch. Ex Poir.). Bangladesh Res. Pub. J. 11(3), 233-236.

Restrepo, S.J.A. y C.F.A. Vallejo. 2008. Caracterización molecular de introducciones colombianas de zapallo $\mathrm{Cu}$ curbita moschata. Acta Agron. 57(1), 9-17.

Restrepo, S.J.A. 2015 Heterosis y habilidad combinatoria en función de la endogamia para el rendimiento y características del fruto de zapallo Cucurbita moschata Duch. ex Poir. Tesis de doctorado. Facultad de Ciencias Agropecuarias, Universidad Nacional de Colombia, Palmira, Colombia.

Rezende, G.S.P. y C.L. Souza-Junior. 2000. A reciprocal recurrent selection procedure outlined to integrate hybrid breeding program in maize. J. Genet. Breed. 54, 57-66.

Ruiz, E., A. Sigarroa y J.A. Cruz. 2004. Análisis dialélico del rendimiento y sus principales componentes en variedades de calabaza (Cucurbita moschata Duch.) I. Tabla dialélica de Griffing. Rev. Biol. 18(1), 65-73.

SAS®. 2012. SAS/STAT Guide for personal computers. Versión 9.4. SAS Institute Inc., Cary, NC, USA.

Toro, S.S. 2009. Heterosis y habilidad combinatoria entre poblaciones seleccionadas de (Cucurbita moschata Duch. Ex Poir). Tesis de maestría. Facultad de Ciencias Agropecuarias, Universidad Nacional de Colombia, Palmira, Colombia. 